\title{
Neurocognitive Adverse Effects of Anesthesia in Adults and Children: Gaps in Knowledge
}

\author{
Christopher G. Ward ${ }^{1}$ - Roderic G. Eckenhoff ${ }^{2}$
}

Published online: 20 April 2016

(C) Springer International Publishing Switzerland 2016

\begin{abstract}
Numerous preclinical and clinical studies investigating the neurodevelopmental and neurocognitive effects of exposure to anesthesia and the combination of anesthesia and surgery have demonstrated histopathological and both temporary and long-term cognitive and behavioral effects at the extremes of the human age spectrum. Increasing coverage in the lay press for both our youngest and oldest patient populations has led to heightened concerns regarding the potential harmful side effects of almost all commonly used anesthetic drug regimens. Although the majority of information regarding anesthetic risks in the developing brain derives from preclinical work in rodents, research involving the aged brain has identified a well-defined postoperative cognitive phenotype in humans. While preclinical and clinical data appear to support some association between anesthesia and surgery and the development of detrimental cognitive changes in both the developing and the aged brain, correlation between anesthesia and surgery and poor neurological outcomes does not imply causation. Given this information, no single anesthetic or group of anesthetics can be recommended over any other in terms of causing or preventing negative neurocognitive outcomes in either population. This review summarizes the growing body of preclinical and clinical
\end{abstract}

Christopher G. Ward

wardc3@email.chop.edu

1 Department of Anesthesiology and Critical Care, Children's Hospital of Philadelphia, 3401 Civic Center Blvd, Philadelphia, PA 19104, USA

2 Department of Anesthesiology and Critical Care, Perelman School of Medicine at the University of Pennsylvania, Philadelphia, PA, USA literature dedicated to the detrimental effects of anesthesia on both the developing and the aging brain.

\section{Key Points}

Many preclinical studies have demonstrated detrimental effects from anesthetic and sedative drugs on both the immature and the aging brain, but the clinical manifestation of these exposures is only hypothesized for children and is better defined in the elderly population with regards to a long-term cognitive consequence.

Whereas baseline cognitive performance and specific biomarkers in the elderly appear to be possible predictors of the development of perioperative neurocognitive decline, thus suggesting the possibility of pre-existing vulnerabilities playing a large factor, no similar vulnerability has been identified in the developing brain at this time.

No single anesthetic or group of anesthetics can be recommended over any other in terms of causing or preventing negative neurocognitive outcomes in either population.

\section{Introduction}

The possible detrimental effects of anesthesia on the developing and aged brain have been increasingly discussed in both the medical literature and the lay press over the past 2 decades, but researchers published concerns 
regarding this topic over 60 years ago-after more than a century of routine clinical use. For example, personality changes were noted in young children after receiving vinyl ether, cyclopropane, or ethylchloride for otolaryngological surgery, although the authors were careful not to implicate the anesthetic [1]. Only 2 years later, an article appeared suggesting that anesthesia might have "adverse cerebral effects" in the elderly [2]. US Center for Disease Control data from 2010 indicate that 2-6 million children under the age of 15 years and roughly 15-20 million patients over the age of 65 years undergo surgery and anesthesia in the USA each year [3,4]. Although patients over the age of 65 years represent only $13 \%$ of the US population, they undergo $20 \%$ of all surgical procedures [5]. A growing body of laboratory, animal, and clinical evidence suggests that general anesthetics may be neurotoxic to both young and aging brains, perhaps more markedly in subsets of the population at risk of developing neurocognitive impairments. Many preclinical studies have demonstrated detrimental effects from anesthetic and sedative drugs on both the immature and the aging brain, but the clinical manifestations of these exposures is only hypothesized for children and better defined in the elderly population with regards to a long-term cognitive consequence. In addition, there is increasing interest in the potential relationship between exposure to general anesthesia and its possible effect on the onset and progression of Alzheimer's disease. Thus, the literature suggests an overall concern that both our youngest and our oldest patients are at increased risk of anesthetic neurotoxicity. In this review, we attempt to summarize the most current data in both animal and human studies related to the topic of anesthesia-associated neurotoxicity as well as address how the research addressing the immature and aged brain has developed over the years. While this review focuses largely on studies of anesthesiainduced neurotoxicity, it must be emphasized that numerous other studies have demonstrated that anesthetics can also induce organ (brain and heart) protection [6, 7]. Since the protective effects of anesthesia are beyond the scope of this review, we do not address them further.

\section{Pediatric Preclinical Concerns}

In addition to the early possible connection between childhood exposure to anesthetics and long-term neurological deficit, researchers in the 1970s and mid-1980s became concerned about the effects of a long-term occupational exposure to anesthetics during pregnancy on the unborn fetus. But it was not until the turn of the twenty-first century that preclinical studies more closely resembling pediatric anesthesia practice commenced [8,9]. A seminal study demonstrated that widespread neuronal degeneration followed exposure to ketamine in neonatal rats [10]. These findings were rapidly followed by a rodent study demonstrating increased neuronal apoptosis and long-term impaired neurocognitive function following neonatal exposure to a combination of midazolam, nitrous oxide, and isoflurane [11]. These studies have now been confirmed by numerous laboratories for almost all routinely used sedatives and anesthetics in over 250 studies using different animal models, including rodents, hamsters, guinea pigs, and rhesus monkeys (see Ward and Loepke [12] or Lin et al. [13] for a comprehensive review). While many of the initial preclinical studies established that the anesthetic exposure for developing animals had to be within a limited time frame early during the brain maturation period, more recent research has shown that anesthetic neurotoxicity risk extends beyond this window [14, 15]. However, it is important to note that a correlation of neonatal brain cell death with long-term abnormalities in neurological function and brain structure has been harder to demonstrate.

Despite the growing accumulation of preclinical animal data that anesthetic drugs pose a harm to both the immature and the developing brain, the topic remains highly debated $[16,17]$. For example, as mentioned above, the widespread neuronal damage demonstrated in different histopathological studies immediately following an anesthetic exposure has not correlated well with subsequent assays of behavior or cognition [18-20]. Further, anesthesia in rodents is accompanied by physiological changes that are difficult to monitor or control. The vast majority of studies in rodents monitored inhaled anesthetic and oxygen concentrations as well as temperature of the rodents and reported the results of a terminal blood gas sample at the end of the exposure, but only one study also measured respiratory rate, blood pressure, and heart rate throughout the exposure to better control the effects of anesthetic agents on physiologic parameters [18, 21]. To address this problem, and to improve translation in general, primate studies are underway that have begun to document extensive neuronal cell death following exposure to isoflurane and propofol [22, 23]. In addition, learning and motivational impairment has been observed in non-human primates exposed to $24 \mathrm{~h}$ of ketamine early in life [24]. Although at this time there are insufficient primate studies to report any generalizations, the presence of conflicting findings should not be surprising, given the variations possible. There may exist differences in the response to anesthetics related to species, anesthetic dose and duration, anesthetic class, drug combinations, and/or rate of plasticity and neurological repair, and behavioral/cognitive assays used. Returning to the example of ketamine, one study reported that a single dose of $50 \mathrm{mg} / \mathrm{kg}$ in newborn mice resulted in abnormal behavior and impaired learning and memory acquisition in adolescence [25]. Yet, another mouse study concluded that 
ketamine $40 \mathrm{mg} / \mathrm{kg}$ did not result in any neurobehavioral abnormalities [26]. Thus, the presence of sharp threshold effects complicate further interpretation.

It should also be noted that, in the majority of these studies, the animals were given anesthetic or sedative drugs without any painful stimulus. Regrettably, few of the hundreds of studies looking at anesthetic neurotoxicity have coupled the anesthetic exposure with surgery, in large part due to the difficulty and safety of performing surgery in a neonatal rodent. Nevertheless, some pediatric practitioners and researchers believe that painful stimuli may modify or even prevent the harmful effects of anesthetics on the developing brain. For example, one study showed that rats experiencing pain alone resulted in neuronal excitation and cell death, but that ketamine inhibited this effect [27]. Unfortunately, especially with smaller animal models, it remains difficult, if not impossible, to maintain physiological stability or even monitor the hemodynamic consequences of the anesthetic exposures alone, let alone in combination with surgery. This frequently leads to a degree of animal mortality (often unreported) that is far greater than what is seen clinically [28].

\subsection{Proposed Mechanisms of Anesthetic Neurotoxicity in the Developing Brain}

While the search for a phenotypical presentation for the possible effect of anesthesia exposure on the developing brain continues to vex researchers, the possible mechanisms of such an effect are beginning to become more apparent. A recent meeting of experts on this topic concluded that two critical factors determine anesthetic neurotoxicity: the stage of brain development at the time of exposure, and the extent of the anesthesia exposure, both in total cumulative anesthetic dose and the frequency of the exposure [29]. Animal studies initially focused on the observation of increased anesthesia-induced neuroapoptosis. This was demonstrated to occur in both the intrinsic and the extrinsic apoptotic pathways and hypothesized to occur during the peak period of synapse formation in the developing brain [14]. Other studies have shown impaired synaptic development and dendritic architecture changes following anesthetic exposure during this same synaptogenesis period [30]. Confounding the translation of these developmental windows of vulnerability from animals to humans is the issue that the peak period of synaptogenesis does not occur at the same time in all brain regions, even within the same species. Therefore, in animal models, different areas of the brain are susceptible to detrimental effects at different stages of development [15]. Studies concentrating on anesthetic effects on neurogenesis in the hippocampus have shown a loss of stem cells and reduction of dentate gyrus neurogenesis [31]. Further studies examining neurotrophic factors such as brain-derived neurotrophic factor (BDNF) demonstrate both isofluraneand propofol-induced reductions in synapse density in the developing hippocampus [32]. Anesthetics that promote $\gamma$ aminobutyric (GABA) receptor activation in the immature brain cause disturbances in mitochondrial membrane potential, which leads to neuronal dysfunction and death [33]. Isoflurane exposure in both cell culture and animal studies has also been shown to impair glial cytoarchitecture in immature astrocytes, which may result in dysfunctional morphological development and proliferation that may influence behavior, learning, and memory later in animal development $[34,35]$. The role of surgery and inflammation is likely an additive if not synergistic propagator of anesthesia-induced developmental neurotoxicity [36]. A key pro-inflammatory factor, interleukin-1-beta, which is elevated during surgery, increases GABA receptors on the surface of hippocampal neurons and may lead to an increase in neurotoxicity associated with these receptors [37]. The role of inflammation in the developing brain is one of the few similarities that is discussed in regards to cognitive dysfunction in the aged brain.

\subsection{Drug Classes Associated with Anesthetic Neurotoxicity in the Developing Animal Brain}

Despite the ongoing debate over the possible effects of anesthetic and sedative drugs on the developing brain, even in preclinical studies, most types of anesthetic drugs appear to have an effect histopathologically and/or on learning and behavior. Although the understanding of the exact molecular mechanisms of how general anesthetic and sedative drugs work to render a patient unconscious or sedated is unknown, the majority of the drugs commonly used in practice either potentiate A-type GABA receptors, inhibit $N$-methyl-D-aspartate (NMDA) glutamate receptors, or agonize alpha-2 receptors. Overlap is possible in some instances. Ethanol has similar effects on NMDA and GABA receptors, so much of the early work in this field attempted to link possible anesthetic effects on the developing brain to those observed in fetal alcohol syndrome $[38,39]$. Anesthetic and sedative drugs can also interfere with neurotransmitter signaling involving acetylcholine, serotonin or AMPA (alpa-amino-3-hydroxy-5-methyl-4isoxazolepropionic acid) or modulate neuronal activity and homeostasis via actions on sodium, potassium, or calcium channels [40]. Opioids, frequently given with general anesthetics clinically, do not act on any of the above receptors, but do produce presumably detrimental effects on the developing brain [41]. Despite this, opioids have not yet received the attention that other anesthetic and sedative drugs have received in either the field of anesthesia or in the lay press [42, 43]. The mechanisms by which 
glutamatergic or GABAergic drugs produce developmental toxicity remain unclear, but excitotoxicity in both cases has been proposed. While counter-intuitive, excitotoxicity for GABAergic drugs may precede the developmental reversal of the chloride gradient [44].

\subsubsection{N-Methyl-D-Aspartate (NMDA) Antagonists}

Glutamate is one of the most common excitatory neurotransmitters in the mammalian central nervous system. Ketamine and nitrous oxide both inhibit NMDA-type glutamate receptors and are routinely used in clinical anesthesia and sedation practice [45]. Xenon, a far less frequently used inhalation agent because of its high cost, also inhibits NMDA-type glutamate receptors amongst other actions [46]. As mentioned above, early studies demonstrated widespread degeneration of brain cells following the administration of ketamine in newborn rat pups [10]. Although many studies have since replicated these findings, it remains unknown whether doses of up to 75 $\mathrm{mg} / \mathrm{kg}$, doses required for small rodents and monkeys because of their increased anesthetic requirements, can be compared across species to the significantly smaller doses (of $1-2 \mathrm{mg} / \mathrm{kg}$ ) used clinically in humans. Although a single ketamine $50 \mathrm{mg} / \mathrm{kg}$ exposure in newborn mice led to abnormal behavior and impaired learning in juvenile animals, neonatal rats given ketamine $5 \mathrm{mg} / \mathrm{kg}$, approaching a human clinical dose, injected daily for 4 days, had no gross neurobehavioral abnormalities when tested as adults. Interestingly, this lower dose of ketamine prevented neuronal degeneration caused by repetitive painful stimulation in these rodents [27]. Exacerbation of the neuroapoptotic effects of ketamine by co-administration of GABAergic drugs, such as midazolam, thiopental, or propofol, common practice during clinical anesthesia and sedation, has also been demonstrated in animal models [47, 48].

Few studies have examined the effects of the NMDA antagonists nitrous oxide or xenon on brain development. Xenon alone had deleterious effects in one study [49] and no effect in another [50], whereas nitrous oxide as a sole agent, even under hyperbaric conditions, did not increase neuronal cell death [11]. However, when xenon was administered concurrently with isoflurane, it reduced some of the neurotoxic effects of isoflurane [41], whereas coadministering nitrous oxide with isoflurane exacerbated the neurodegenerative properties of isoflurane [11].

\subsection{2 $\gamma$-Aminobutyric $(G A B A)_{A}$ Agonists}

GABA is the most prevalent inhibitory neurotransmitter in the adult central nervous system (CNS), but it is important to note that GABA is excitatory during early brain development [44]. The basis is a reversal of the cellular chloride gradient due to developmental expression of specific chloride transporters [51]. The implications of this developmental switch on anesthetic-induced neuronal cell death remain unclear. Regardless, commonly used drugs with at least some GABAergic activity such as isoflurane, sevoflurane, desflurane [52], pentobarbital, clonazepam, diazepam [53], midazolam [48], choral hydrate [54], and propofol [55] have all been shown to cause neuronal cell death in immature animal species. Although it is clear that timing of the exposure is critical in the induction of 'neurotoxicity', studies have not yet specifically addressed this timing with respect to timing of the chloride gradient reversal. This would be important to discern between an excitotoxicity mechanism, or a neuronal quieting mechanism.

\subsubsection{Alpha2-Agonists}

Dexmedetomidine has been gaining increasing importance in pediatric clinical practice for its desirable ability to sedate children without comprising the airway or causing other unwanted side effects associated with other anesthetic and sedative drugs. The few studies of dexmedetomidine show limited to no neurotoxic effects when given alone. When given to neonatal rodents with isoflurane, neuroapoptosis was reduced, and subsequent learning ability improved [56]. Another group demonstrated that dexmedetomidine had less of a pro-apoptotic effect on the neonatal rat brain than ketamine [57]. As a result of this limited preclinical evidence, dexmedetomidine has received increased attention as a potentially 'safe' anesthetic in children, despite it being considered an 'incomplete' anesthetic in that it does not render a patient completely unconscious, meaning other drugs, such as an opioid, are necessary.

\subsubsection{Opioids}

Although opioids are rarely used as the sole agents for pediatric anesthesia and sedation, they are often administered in conjunction with the above-mentioned drugs. When used as a 'balanced anesthetic technique' in preclinical trials, their co-administration can significantly reduce dose requirements for drugs with known neurotoxic effects and potentially mitigate any negative effects. Narcotics have also been demonstrated to improve clinical outcomes in pediatric cardiac surgery [58]. However, numerous preclinical studies of opioids in developing animals have also shown deleterious effects. Neonatal rat exposure to morphine has caused apoptosis in selective brain regions [41]. Further, perinatal exposure of rodents to opioids was demonstrated to induce long-term behavior and learning impairments into adulthood [59]. 


\subsection{Human Studies}

Assessing the above observed neurotoxicity phenomenon in children via randomized, placebo-controlled clinical trials of anesthesia/sedation with surgery and the histological examination of post-exposure brains in healthy children is unfeasible for obvious ethical reasons. Numerous factors (differing species, drug doses and durations, lack of physiologic monitoring and control) complicate the translation of the aforementioned animal studies regarding anesthetic neurotoxicity. Moreover, difficulties in translating developmental time point equivalents between species, as well as scaling drug exposure durations to developmental timing or life span have contributed to considerable uncertainty with how (or if) to translate to the human patient $[60,61]$ Thus, patient-oriented studies are an absolute necessity. However, here too, the cohort studies investigating neurodevelopmental outcome following anesthesia exposure early in life are confounded by the increased severity of illness and coexisting congenital abnormalities that require surgical intervention versus medical management of control subjects [62]. In particular, as the involvement of neuroinflammation becomes a more widely recognized influence on the developing nervous system, the significance of common medical conditions in neonates requiring surgery such as necrotizing enterocolitis cannot be convincingly separated from the influence of the anesthetic exposure [63].

\subsubsection{Epidemiological Studies Examining Long-Term Neurodevelopmental Effects of Surgery with Anesthesia}

Given the repeated and overwhelming evidence of anesthetic influences on the developing brain in animal models, several epidemiological studies have attempted to address any possible effects of surgery and anesthesia on different neurocognitive and behavioral outcomes using cohorts of largely healthy individuals. Since early animal research that proposed a vulnerable period during rapid synaptogenesis, a period known as the 'brain growth spurt,' most studies concentrated on anesthetic exposures in children aged under 3-4 years. Another factor driving epidemiological studies to focus on this age group and younger was an influential 2009 study of children born between 1976 and 1982 in Rochester, MN, USA [64]. In this study, children who underwent two or more surgeries with anesthesia or had a combined anesthesia time greater than 120 min prior to the age of 4 years had a twofold increased risk of developing a learning disability later in life. A single exposure to anesthesia in this cohort of more than 5300 children prior to the age of 4 was not associated with later learning disability. While this study has helped guide many of the retrospective studies that have followed, the significance of its findings have been questioned because of changes in anesthetic practice since 1980, notably, the introduction of routine pulse oximetry and capnography and the elimination of halothane and more limited use of nitrous oxide. The school records including the 'learning disability' diagnosis were also limited in that an unknown range of disabilities and domains existed for the same diagnosis over different ages.

To further refine this finding, these authors performed a subsequent analysis from the same population-based retrospective birth cohort, where 350 children exposed to anesthesia before the age of 2 years were matched with 700 children not exposed to anesthesia [65]. This time, the investigators more carefully defined 'learning disability' as the need for individualized education for speech and language impairment, as well as abnormal scores on achievement tests. Once again, children receiving only a single anesthetic prior to the age of 2 years were not different from controls on group-administered achievement tests or the need for individualized speech and language interventions, but the group of multiple anesthetic exposures had significantly lower test scores and higher need for intervention. These results remained after adjusting for sex, birth weight, gestational age at birth, mother's education, and severity of illness. The investigators also used this birth cohort to examine the effect of perinatal exposure to general anesthesia for cesarean section. General anesthesia for cesarean section was not associated with an increased risk for subsequent diagnosis of a learning disability in almost 200 patients as compared with neuraxial anesthesia. However, and interestingly, the neuraxial group had a lower risk of learning disability than children who underwent spontaneous vaginal deliveries [66]. A subsequent analysis of a larger cohort did not detect any differences in learning disability between those having spontaneous vaginal delivery and those having vaginal delivery under neuraxial labor analgesia.

In studies using the New York State Medicaid database, 383 children undergoing a single anesthetic exposure for an inguinal hernia repair prior to the age of 3 were compared with over 5000 age-matched children who underwent no surgery [67]. These investigators found an increased risk of being diagnosed with developmental or behavioral disorders later in life but, in this case, only following a single anesthetic. Given that their data derived from the New York Medicaid database, the influence of economic and social influences (known confounders on learning and development) could not be excluded [68]. Another study by this group of researchers also found an association between two or more anesthetic exposures prior to the age of 3 years and developmental or behavioral disorders [69]. Of interest, a smaller sub-analysis of matched sibling pairs could 
not detect an association, a fact that led to the PANDA (Pediatric Anesthesia and Neurodevelopment Assessment) study. This study used an ambidirectional cohort design, performing prospective neuropsychological assessment in exposed-unexposed sibling pairs aged 6-11 years in which one sibling received anesthesia for an inguinal hernia repair prior to the age of 3 years. Although the results of this study are yet to be published, it is anticipated they will be released in the next few months [70].

In a study of monozygotic twins born between 1986 and 1995 from the Netherlands Twin Register [71], in which one twin received anesthesia prior to the age of 3 years and the other did not, no difference in school performance between the discordant twins was detected. However, twins both exposed to anesthesia before the age of 3 years did have lower educational achievement than unexposed twins. This finding suggests that other factors, such as genetic predisposition or social and economic factors may have a greater contribution to school performance than anesthetic exposure.

Further support for this notion comes from a Swedish study that investigated national standardized testing results for approximately 2 million children at the age of 16 years [72]. Almost 35,000 children in this cohort had one anesthetic exposure between birth and 4 years of age and had no further hospitalizations or surgery. Unexposed controls were matched on sex, parity, and year and month of birth. Although the study found that children with one exposure prior to the age of 4 years had a $0.47 \%$ lower average score when tested at age 16 on the national exam, further examination of this cohort demonstrated a far greater influence from factors other than surgery and anesthesia exposure on test scores. For example, males scored $9.87 \%$ lower, children born in December (young for their grade) versus January (old for their grade) had $5.31 \%$ lower scores, and children whose mothers did not attain a college degree had $9.92 \%$ lower average scores. Two Danish studies utilized a birth cohort born between 1986 and 1990 and concentrated on only outcomes following inguinal hernia repair or pyloromyotomy [73, 74]. Despite both patient populations having an exposure to anesthesia and surgery before the age of 1 year, test scores did not differ between either group and those in the unexposed general population. Although these results are reassuring, both Danish studies acknowledged that children who had experienced surgery as infants were less likely to sit for the examination, the interpretation of which depends on the underlying reasons.

While a surgical and anesthetic exposure appears to have a minimal effect on group-administered standardized tests, perhaps this outcome lacks the sensitivity to capture the consequence of surgery and anesthesia on the developing brain. The Raine cohort, a birth cohort born between
1989 and 1992 in Western Australia and initially developed to test the effect of maternal ultrasounds on the developing fetus, has been followed with serial neuropsychological evaluations that go beyond school records. Although no difference in group-administered academic tests was detected in those receiving surgery and anesthesia exposure prior to the age of 3 , the authors found measurable differences using the directly administered neuropsychological tests and billing coded clinical disorders [75]. In another study, albeit very small, 28 children aged 6-11 years who had undergone a procedure requiring general anesthesia prior to the age of 1 year were compared with age- and sex-matched children who received no surgery. The investigators found no difference between exposed and unexposed in familiarity, IQ, and Child Behavior Checklist scores, but did find that exposed children had significantly lower color recollection scores, which suggests anesthesia and surgery in infancy may impair recollecting associative information later in life [76].

Given the difficulty of determining what clinical outcomes are important to define the still unknown phenotype following surgery and anesthesia in the developing brain, others have turned to neuroimaging to detect differences. One such study examined healthy participants of a language developmental study in individuals aged 5-18 years who had surgery with anesthesia prior to the age of 4 year [77]. Although both groups scored within population norms in regards to neurocognitive assessments (Oral and Written Language Scales and the Weschsler Adult Intelligence Scales or the Wechsler Intelligence Scale for Children), previously exposed children scored lower in listening comprehension and performance IQ. More importantly, these decrements were associated with lower gray matter density in the occipital cortex and cerebellum, but not in regions identified as susceptible in the many animal studies.

Studies of infants with complex congenital heart disease also exist, but the neurodevelopmental outcomes they measured were strongly confounded by the severity of the illness. One study using the Bayley Scales of Infant Development at 2 years of age found no developmental delay after anesthesia and surgery, but did find an association with ventilator days and older age at surgical correction for congenital heart disease [78]. However, a recent follow-up study demonstrated reduced visual motor integration skills with higher cumulative benzodiazepine doses and lower performance IQ scores with previous exposure to greater chloral hydrate doses [79]. A similar study involved 60 neonates undergoing complex congenital heart surgeries with Bayley Scales of Infant Development scores at 12 months of age as the primary outcome. They found an association between decreased scores and increased volatile anesthetic exposure, and increased length of stay in the 
intensive care unit [80]. It is difficult to attribute such differences in cognitive outcomes to the drugs used per se, given the enormous physiological and psychological trespass these infants experience. It is nonetheless reassuring that differences are relatively small and often difficult to detect, suggesting a robust process of neuroplasticity. The premature infant is another vulnerable patient population. Nearly 100 surviving premature infants, born between 22 and 32 weeks post-conceptual age who were treated with sedatives and analgesics were compared with about 1200 non-sedated children [81]. No difference was found in disability rates at a 5-year follow-up assessed using the Kaufman Assessment Battery for Children.

\subsubsection{Prospective Human Study}

Preliminary results from the first prospective study to examine the effect of general anesthesia and surgery on neurodevelopmental outcome have been released [82]. The GAS (General Anesthesia compared to Spinal anesthesia) trial is an international assessor-masked randomized controlled equivalence trial that enrolled infants younger than 60 weeks postmenstrual age who were born at $>26$ weeks' gestation and who presented for inguinal hernia repair. They were randomly assigned to receive either awake regional anesthesia or a sevoflurane-based general anesthetic. Although the primary outcome of the trial will be the Wechsler Preschool and Primary Scale of Intelligence-third edition (WPPSI-III) Full Scale Intelligence Quotient score at the age of 5 years, the authors reported the secondary outcome of the Bayley Scales of Infant and Toddler Development III assessed at 2 years. They found that an average sevoflurane exposure of around $1 \mathrm{~h}$ in infancy did not cause an increased risk of adverse neurodevelopmental outcome compared with awake regional anesthesia. While these 2-year outcomes are reassuring, the 5 -year cognitive outcomes will provide a more comprehensive assessment of the possible effects of a single, relatively short anesthetic and surgical exposure.

In 2014, the International Anesthesia Research Society and the US FDA convened a group of experts in anesthesia, pediatric medicine, neuroscience, and patient safety to consider the mixed evidence from preclinical and especially clinical data related to any possible detrimental effect from anesthesia and surgery on the developing brain. Wishing to address the concerns of both healthcare providers and parents, this group, known as SmartTots, recently released the Consensus Statement on the Use of Anesthetic and Sedative Drugs in Infants and Toddlers. This consensus is endorsed by almost 20 stakeholder organizations, including the American Society of Anesthesiologists, the American Academy of Pediatrics, the Society for Pediatric Anesthesia, and the FDA. It states that "(b)cause there is not enough information about the effects of anesthetic drugs on the brains of young children, it is not yet possible to know whether use of these medicines poses a risk, and if so, whether the risk is large enough to outweigh the benefit of the planned surgery, procedure or test" [83].

\section{Postoperative Cognitive Decline}

Approximately $15 \%$ of patients aged $>60$ years experience objectively measured decreases in cognitive function, now known as perioperative neurocognitive disorder (PND) following an episode of anesthesia and surgery [84]. PND includes postoperative delirium, as well as a spectrum of cognitive findings and complaints of varying duration, and is more useful terminology than postoperative cognitive dysfunction (POCD) [85]. Although the exact causes remain unknown, the risk factors include advanced age, lower educational achievement, and pre-operative cognitive disorders [86]. The cognitive changes after anesthesia and surgery are usually temporary, with normal function returning within a few days, but may remain for weeks, months, or even years. Many PND changes are mild and only detected by specific neuropsychological testing following anesthesia and surgery, but subjective complaints of memory loss, impaired higher-level cognitive function, issues with fine motor coordination, dementia, depression, delirium, or psychomotor derangement have been reported [87, 88]. Many of these alterations in cognitive function correlate with increased hospital length of stay, risk of other complications such as in-hospital falls, and increased cost.

Early research attributed much of the PND observed in elderly patients to cardiopulmonary bypass (CPB) during cardiac surgery, but more recently has been demonstrated to also occur following non-cardiac surgery [89]. The incidence of PND in patients aged $>65$ years after noncardiac surgery has been reported as high as $26 \%$ at 1 week following surgery, $10 \%$ at 3 months [90], $5 \%$ at 6 months, and $1 \%$ at 12 months [91]. However, PND has been reported following cardiac surgery to be as high as 53, 36,24 , and $42 \%$ at discharge, 6 weeks, 6 months, and 5 years, respectively [92]. It is important to note that the understanding and therefore the definition of PND continues to evolve towards standardization. Thus, the large variance in incidence found in the current literature is likely to decline [93].

The aging brain undergoes neuron loss, decreased rates of neurogenesis and synaptogenesis, and the accumulation of potentially toxic byproducts secondary to declining organ function. This diminished reserve is thought to increase vulnerability to any number of neural insults. The neurotoxic effects of anesthesia are hypothesized to result 
from direct effects from the drugs (such as from changes in calcium homeostasis) [94], enhancement of endogenous neurodegenerative mechanisms (increased production of $\beta$ amyloid or increasing hyperphosphorylation of tau protein) [95, 96], amplification of neuroinflammation generated by surgically induced systemic inflammation [97], modulation of synaptic plasticity [98, 99], suppression of stem cell proliferation or differentiation [100], or a weakened bloodbrain barrier (BBB) function [101, 102]. As with anesthesia effects in the developing brain, the confounding effects of surgery and associated diseases requiring surgery in the aged brain strongly influence neurodegenerative outcomes. In addition, neurodegenerative disorders in the elderly population are known to have a long asymptomatic phase, but during this time the brain is more vulnerable to superimposed insults. Similarly, even if symptomatic, there exists a bias against diagnosis due to social stigma of the label 'dementia.' Whereas the ultimate societal and clinical implications of anesthesia and surgery in the young developing brain remain to be determined, it is very clear that PND is associated with an increased dependency on social support, premature withdrawal from the labor market, and increased mortality [103]. Further, whereas a specific susceptible subgroup of neonates, infants, or children has yet to be identified, the pre-existing vulnerabilities (risk factors) for PND are becoming clear, thereby allowing future research to focus on subgroup analysis to identify these patient populations and/or devise mitigating strategies [87].

Though the mechanisms of PND are unknown, its similarity to Alzheimer disease (except for the reversibility) has allowed related upstream hypotheses: amyloidopathy, tauopathy, calcium dysregulation, mitochondrial dysfunction, and BBB dysfunction [104]. Many of these might coexist, and a possible common denominator appears to be amplified neuroinflammation, which is likely a common downstream pathway for producing synaptic and neuronal damage [105, 106]. Many anesthetics have been shown to interact with each of the upstream pathways, while surgery and systemic inflammation interact with the downstream pathway, possibly making this a complicated interplay of numerous, diverse, overlapping, and separate pathways [107].

\subsection{Preclinical Evidence for Perioperative Neurocognitive Dysfunction}

Inhaled anesthetics have been demonstrated to cause damage in various types of tissues and cells, including primary cortical and striatal neurons, neuroglioma cells, and hippocampal slices. Various in vitro models have also shown 'toxic' responses to anesthetic gases. These in vitro studies demonstrated that inhaled anesthetics produce effects in isolated proteins, cells in culture, and brain slices consistent with increased cellular stress via recognized pathways of neurodegeneration [108]. Most of the work done with inhalational agents such as isoflurane and sevoflurane caused increased $\beta$-amyloid production and aggregation and cellular apoptosis [108, 109]. Importantly, cells transfected with genes associated with Alzheimer disease are more sensitive to these presumably detrimental effects of anesthetic [110]. Of note, many of these studies used tumor cells rather than primary neurons, as well as cells already under stress (serum starved), so the translation even to animals remains necessary but uncertain.

\subsection{Animal Studies of Perioperative Neurocognitive Disorder (PND)}

Studies in animals allow anesthesia to be considered separately from surgery, but it is important to emphasize that, as in the young animals, the physiologic monitoring and ability to correct the often detrimental effects of an anesthetic on animal physiology is suboptimal at best. Thus, while anesthesia can be separated from surgery, direct effects of the anesthetic cannot be reliably separated from the many physiologic changes that anesthetics produce, many of which are plausible contributors to the neuropathology under consideration. Although results of anesthesia-only exposures have been somewhat inconsistent, in general, anesthetic exposure by itself in adult wildtype animals does not cause durable cognitive deficits. On the other hand, in aged rodent models, exposure to anesthetics alone, notably isoflurane, has caused persistent memory impairment, accompanied by histopathological evidence of amyloidopathy and neuronal injury [111, 112]. When introducing additional vulnerabilities in the form of Alzheimer-associated genes, both halothane and isoflurane have been shown to enhance the histopathology associated with neurodegeneration [112]. In general, investigators have noted that the degree of injury, and even the direction of effect, is anesthetic dose and time dependent [113, 114], but some rodent studies have shown that even brief exposures to anesthesia can induce tau hyperphosphorylation, probably because of hypothermia [115]. Thus, to summarize a very large body of literature from over the last decade, volatile anesthesia alone has either a minor or no effect on rodent learning and memory in the absence of other susceptibility factors such as age or genetic alteration. Even in the presence of these factors, the effect remains small [97, 110].

In the last few years, investigators have begun to add surgery to their animal models, both in the recognition that anesthesia alone seems relatively innocuous and to improve the translatability of the model. The surgery has included tibial osteoplasty [116], splenectomy [99], cecal 
ligation [117], and excision and partial hepatectomy [118], and has generally been considered 'clean' (e.g., non-septic). Defects in rodent learning and memory were amplified [119], especially as a function of age and in the context of Alzheimer-susceptibility transgenes [119]. In the latter example, cognitive effects of anesthesia alone were reversible after a few weeks, but the addition of surgery made the defects essentially irreversible [116]. The mechanism underlying the exacerbation by surgery was initially proposed to be neuroinflammation, a notion that is now generally accepted. For example, many studies have demonstrated elevated interleukin (IL)-6 and tumor necrosis factor (TNF) $\alpha$, both in blood and in brain tissue, along with activated microglia $[116,120]$. Mitigation with anti-TNF antibodies or with other anti-inflammatory strategies generally reduced both histopathology and cognitive changes. Another study suggested that endogenous anti-inflammatory pathways (IL-4, IL-10) were rendered dysfunctional in neurodegenerative disease, allowing an unopposed pro-inflammatory process to damage synapses and neurons [121]. How systemic inflammation is transduced into neuroinflammation is still unclear, but several plausible pathways exist. It is likely that the systemic inflammatory response results in a leaky BBB and neuroinflammation [103]. This is the probable mechanism for the 'sickness' behavior observed during infection or after lipopolysaccharide (LPS) injection [122]. It is also possible that cholinergic afferents from the gut activate central inflammatory pathways in the absence of BBB dysfunction [123].

It is also becoming clear that neuroinflammation can be modulated by anesthetic choice. For example, microglia in culture exhibit a dramatically different response to activation by LPS when exposed to different anesthetics. Sevoflurane enhances the secretion of proinflammatory cytokines, propofol completely abolishes it, and isoflurane appeared to have little effect [124]. Further, the integrins, a group of proteins mediating leukocyte arrest, migration, and activation, are specifically inhibited by certain anesthetics, notably isoflurane [125]. Finally, anesthetics of many types are well known to have both excitatory and inhibitory effects on a wide array of ion channels, many of which are expressed in leukocytes [126]. Thus, the opportunity for anesthetic choice to influence the innate immune response is particularly rich and also understudied.

Few preclinical studies have appeared that combine a more severe inflammatory insult, such as sepsis, with anesthesia to study the effect on cognitive outcomes. These studies are particularly difficult to conduct, partly due to the complexity of sepsis, the variability in response, and the typically high mortality. Thus, it remains unclear whether the anesthetic choice modulates either mortality or cognitive outcomes in this specific patient population.
In summary, the preclinical data have allowed estimates of effect magnitude of some of the different features inherent to the perioperative experience. Anesthesia alone appears to play only a small role, but when combined with surgery and other susceptibility factors (age, disease transgenes), a marked and durable effect on cognitive outcomes is observed. The mechanism is hypothesized to be an acute neuroinflammatory event superimposed on a chronic smoldering neuropathologic state, accelerating the trajectory of damage and functional decline; however, as discussed in the following section, this is yet to be proven in human studies.

\subsection{Evidence for an Association Between Anesthesia and PND}

Many retrospective and prospective clinical studies have specifically addressed anesthetic type (e.g., regional vs. general) and have consistently been unable to detect a difference in 'POCD' [127, 128]. The widely varying definition of POCD, when patients were tested, and different surgical procedures and inclusion criteria may have contributed to a lack of sensitivity, but this generally agrees with the preclinical data that the influence of the anesthetic itself is small. However, and interestingly, almost all studies are able to detect a considerable incidence in PND, typically $>10 \%$ in older age groups [121]. Another feature found in most studies that measure cognition at more than one time point postoperatively find that the incidence declines over 3-6 months [121]. Whether PND persists beyond a year is still controversial. A few studies have looked for differences in cognitive outcomes between intravenous and inhalational general anesthesia. In one prospective randomized trial in spinal surgery, and with clinical cognitive outcomes at 1 year, investigators found sevoflurane to be associated with an enhanced trajectory toward cognitive impairment [129]. The only other human study to detect a difference between intravenous and inhalational anesthesia was a study of cerebrospinal fluid (CSF) cytokines during and after a fairly minor surgical procedure [130]. Inhalational anesthesia (sevoflurane) was associated with a significantly higher IL-6 level in the CSF after surgery than was intravenous anesthesia (largely propofol) [131]. It is important to note that one recent study following the effect of a single high dose of dexamethasone on outcomes following cardiac surgery failed to demonstrate a reduction in the risk of POCD after cardiac surgery [131]. However, it is not clear that a single dose of even high-dose dexamethasone effectively ablates the inflammatory response, as this study did not measure any indices of inflammation.

In addition to the class of anesthetic, the dose or depth of anesthesia may also modulate the effect on subsequent 
cognition. For example, the recently released Cognitive Dysfunction After Anesthesia trial demonstrated that a general anesthetic guided by the Bispectral index (BIS; a way of controlling dose and depth of anesthesia) had a reduced total anesthetic drug dose as well as a decreased rate of PND at 3 months after surgery [132]. A similar study, albeit with significantly less reduction in anesthetic usage than the previous trial, showed that BIS monitoring led to a reduction in postoperative delirium but not PND at 3 months [133]. Finally, a prospective randomized trial of BIS-guided anesthetic and cerebral oxygenation found a significantly reduced incidence of PND at 3 months and at 1 year in the group where the practitioner was provided with the additional monitoring data [134]. It is important to note that the overall reliability of the BIS monitor to adequately measure has been questioned by members of the anesthesia community [135].

The similarities between symptoms of PND and Alzheimer disease have prompted a number of retrospective studies to look for an association between surgery and incident dementia. In three probably underpowered studies, a significant relationship between surgery and incident Alzheimer disease was not detected, although one detected a relationship between surgery and the age of Alzheimer disease diagnosis, suggesting an acceleration rather than causation [136, 137]. Using more contemporary Alzheimer Disease Center (ADC) databases, another retrospective study explored the impact of surgery, illness, or neither on the trajectory of cognitive decline in the elderly [138]. They were unable to find an association between either surgery or illness with cognitive trajectory, although another more recent study was able to detect a significant relationship between hospitalization and cognitive decline [139]. Two other recent studies examining the effects of anesthesia and surgery using claims data from 1 million insured residents covered under Taiwan's universal health insurance from 2004 to 2007 and 2005 to 2009, demonstrated an increased risk of dementia, particularly in subjects who experienced repeated exposures to general anesthesia, regardless of whether it was an intravenous or inhaled anesthetic technique [140, 141]. Using Alzheimer Disease NeuroImaging (ADNI) data, researchers were able to detect not only a significant relationship between prior surgery and cognitive loss but also a greater loss of hippocampal and cortical grey matter volume as compared with patients who had not undergone surgery [142]. Nevertheless, in a thorough study of Olmstead county residents, researchers were unable to detect any association between surgery and anesthesia with incident dementia [143].

Finally, the similarity between Alzheimer disease and PND has prompted questions as to whether PND is a risk factor for Alzheimer disease. This was addressed in the initial ISPOCD (International Study of Post-Operative
Cognitive Dysfunction) study cohort, and a significant correlation was not detected, partly because of a lack of power [144]. The opposite question, whether a risk of Alzheimer disease could be considered a risk factor for PND has also recently been addressed. For example, patients with cognitive symptoms compatible with mild cognitive impairment were found to be at increased risk for PND, and, second, patients with CSF biomarkers consistent with a diagnosis of Alzheimer disease have a higher risk of PND at 3 months after surgery [145]. An earlier study that examined patients who had total hip/knee-replacement surgeries also noted that pre-operative CSF $\beta$-amyloid-toTau ratios may identify patients at higher risk for cognitive changes after surgery [146]. Highly consistent with the preclinical data mentioned above, these important recent data suggest that the combination of a pre-existing vulnerability with a superimposed neuroinflammatory stress are required to accelerate the trajectory of cognitive decline. In summary, it seems clear that Alzheimer disease and PND are related in some way, and that risk stratification using Alzheimer disease biomarkers and cognitive assessments pre-operatively may predict PND and potentially identify a group of patients in whom mitigation strategies might be useful.

\section{Conclusion}

Although at first glance the weight of the preclinical and clinical data appears to support some association between anesthesia and surgery and the development of detrimental cognitive changes in both the developing and the aged brain, definitive prospective studies in both these areas of research are needed to conclude this with confidence. In both of those two seemingly different patient populations, correlation between anesthesia and surgery and poor neurological outcomes does not imply causation. Given this information, no single anesthetic or group of anesthetics can be recommended over any other in terms of causing or preventing negative neurocognitive outcomes in either population. While baseline cognitive performance and specific biomarkers in the elderly appear to be a possible predictor of the development of PND, thus suggesting the possibility of pre-existing vulnerabilities playing a large factor, no similar vulnerability has been identified in the developing brain at this time. A large part of the difficulty in defining vulnerability in the pediatric population is that a phenotype following exposure to anesthesia and surgery in the developing brain remains to be defined. Anesthetic choice seems to play a minor role in both the young and the old brain. Future research, particularly in the translational and clinical fields, should therefore not only continue to evaluate the mechanisms of the effects of anesthesia and 
surgery on the brain, but also work to identify vulnerable subsets of the population that may benefit from mitigating and/or enrichment strategies to counter any negative effect of anesthesia and surgery. With regards to the developing brain, identifying children who would benefit from early interventions in school and with therapists for language, social, and behavioral and emotional development may be the best avenue, as most retrospective studies thus far appear to show little effect in the general population. In terms of the aging brain, identifying risk factors for PND as well as interventions aimed at maintaining independence and preventing postoperative cognitive decline and dementia will be incredibly beneficial. Regardless, all perioperative healthcare providers, not just anesthesiologists and surgeons, need to be aware of the potential link between anesthesia and surgery and neurocognitive impairments in the developing and the aged brain.

\section{Compliance with Ethical Standards}

Funding No sources of funding were used to assist in the preparation of this study.

Conflict of interest Christopher Ward and Roderic Eckenhoff have no conflicts of interest that are directly relevant to the content of this study.

\section{References}

1. Eckenhoff JE. Relationship of anesthesia to postoperative personality changes in children. Am J Dis Child. 1953;86:587-91.

2. Bedford PD. Adverse cerebral effects of anaesthesia on old people. Lancet. 1955;269(6884):259-63.

3. Sun L. Early childhood general anaesthesia exposure and neurocognitive development. Br J Anaesth. 2010;105(Suppl 1):i61-8.

4. Centers for Disease Control and Prevention. Numbers by procedure category and age [Internet]. 2010. Available from: http:// www.cdc.gov/nchs/data/nhds/4procedures/2010pro4_number procedureage.pdf. Accessed 12 Nov 2015.

5. The American Geriatrics Society. Common surgical procedures [Internet] 2014. Available from: http://www.americangeriatrics. org/gsr/anesthesiology/common_surgical_procedures.pdf. Accessed 12 Nov 2015.

6. Wei H, Inan S. Dual effects of neuroprotection and neurotoxicity by general anesthestics: role of intracellular calcium homeostasis. Prog Neuropsychopharmacol Biol Psychiatry. 2013;47:156-61.

7. Landoni G, Fochi O, Tritapepe L, et al. Cardiac protection by volatile anesthetics. A review. Minerva Anestesiol. 2009;75(5):269-73.

8. Quimby KL, Katz J, Bowman RE. Behavioral consequences in rats from chronic exposure to 10 PPM halothane during early development. Anesth Analg. 1975;54:628-33.

9. Uemura E, Levin ED, Bowman RE. Effects of halothane on synaptogenesis and learning behavior in rats. Exp Neurol. 1985;89:520-9.

10. Ikonomidou C, Bosch F, Miksa M, et al. Blockade of NMDA receptors and apoptotic neurodegeneration in the developing brain. Science. 1999;283:70-4.
11. Jevtovic-Todorovic V, Hartman RE, Izumi Y, et al. Early exposure to common anesthetic agents causes widespread neurodegeneration in the developing rat brain and persistent learning deficits. J Neurosci. 2003;23:876-82.

12. Ward CG, Loepke AW. Anesthetics and sedatives: toxic or protective for the developing brain? Pharmacol Res. 2012;65(3):271-4.

13. Lin EP, Soriano SG, Loepke AW. Anesthetic neurotoxicity. Anesth Clin. 2014;32(1):133-55.

14. Yon JH, Daniel-Johnson J, Carter LB, Jevtovic-Todorovic V. Anesthesia induces neuronal cell death in the developing rat brain via the intrinsic and extrinsic apoptotic pathways. Neuroscience. 2005;135:815-27.

15. Hofacer RD, Deng M, Ward CG, et al. Cell-age specific vulnerability of neurons to anesthetic toxicity. Ann Neurol. 2013;73(6):695-704.

16. Loepke AW, McGowan FX Jr, Soriano SG. CON: the toxic effects of anesthetics in the developing brain: the clinical perspective. Anesth Analg. 2008;106:1664-9.

17. Jevtovic-Todorovic V, Olney JW. PRO: anesthesia-induced developmental neuroapoptosis: status of the evidence. Anesth Analg. 2008;106:1659-63.

18. Loepke AW, Istaphanous GK, McAuliffe JJ 3rd, et al. The effects of neonatal isoflurane exposure in mice on brain cell viability, adult behavior, learning, and memory. Anesth Analg. 2009;108:90-104.

19. Xu H, Liu ZQ, Liu Y, et al. Administration of midazolam in infancy does not affect learning and memory of adult mice. Clin Exp Pharmacol Physiol. 2009;36:1144-8.

20. Liang G, Ward C, Peng J, et al. Isoflurane causes greater neurodegeneration than an equivalent exposure of sevoflurane in the developing brain of neonatal mice. Anesthesiology. 2010;112:1325-34.

21. Disma N, Mondardini MC, Terrando N, et al. A systematic review of methodology applied during preclinical anesthetic neurotoxicity studies: important issues and lessons relevant to the design of future clinical research. Paediatr Anaesth. 2016;26(1):6-36.

22. Brambrink AM, Evers AS, Avidan MS, et al. Isoflurane-induced neuroapoptosis in the neonatal rhesus macaque brain. Anesthesiology. 2010;112:834-41.

23. Creeley C, Dikranian K, Dissen G, et al. Propofol-induced apoptosis of neurones and oligodendrocytes in fetal and neonatal rhesus macaque brain. Br J Anaesth. 2013;110(Suppl 1):i29-38.

24. Paule MG, Li M, Allen RR, et al. Ketamine anesthesia during the first week of life can cause long-lasting cognitive deficits in rhesus monkeys. Neurotoxicol Teratol. 2011;33:220-30.

25. Fredriksson A, Archer T, Alm H, et al. Neurofunctional deficits and potentiated apoptosis by neonatal NMDA antagonist administration. Beha Brain Res. 2004;153:367-76.

26. Rudin M, Ben-Abraham R, Gazit V, et al. Single-dose ketamine administration induces apoptosis in neonatal mouse brain. J Basic Clin Physiol Pharmacol. 2005;16:231-43.

27. Anand KJ, Garg S, Rovnaghi CR, Narsinghani U, Bhutta AT, Hall RW. Ketamine reduces the cell death following inflammatory pain in newborn rat brain. Pediatr Res. 2007;62(3):283-90

28. Sanders RD, Hassell J, Davidson AJ, Robertson NJ, Ma D. Impact of anaesthetics and surgery on neurodevelopment: an update. Br J Anaesth. 2013;110(Suppl 1):i53-72.

29. Jevtovic-Todorovic V, Absalom AR, Blomgren $\mathrm{K}$, et al. Anaesthetic neurotoxicity and neuroplasticity: an expert group report and statement based on the BJA Salzburg Seminar. Br J Anaesth. 2013;111(2):143-51.

30. Briner A, Nikonenko I, De Roo M, et al. Developmental stagedependent persistent impact of propofol anesthesia on dendritic 
spines in the rat medial prefrontal cortex. Anesthesiology. 2011;115:282-93.

31. Zhu C, Gao J, Karlsson N, et al. Isoflurane anesthesia induced persistent, progressive memory impairment, caused a loss of neural stem cells, and reduced neurogenesis in young, but not adult, rodents. J Cereb Blood Flow Metab. 2010;30:1017-30.

32. Head BP, Patel HH, Niesman IR, et al. Inhibition of p75 neurotrophin receptor attenuates isoflurane mediated neuronal apoptosis in the neonatal central nervous system. Anesthesiology. 2009;110:813-25.

33. Sanchez V, Feinstein SD, Lunardi N, et al. General anesthesia causes long-term impairment of mitochondrial morphogenesis and synaptic transmission in developing rat brain. Anesthesiology. 2011;115:992-1002.

34. Culley DJ, Cotran EK, Karlsson E, et al. Isoflurane affects the cytoskeleton but not survival, proliferation, or synaptogenic properties of rat astrocytes in vitro. Br J Anaesth. 2013;110 (Special issue):i19-i28.

35. Lunardi N, Hucklenbruch C, Latham JR, et al. Isoflurane impairs immature astroglia development in vitro: the role of actin cytoskeleton. J Neuropathol Exp Neurol. 2011;70:281-91.

36. Shu Y, Zhou Z, Wan Y, et al. Nociceptive stimuli enhance anesthetic-induced neuroapoptosis in the rat developing brain. Neurobiol Dis. 2012;45:743-50.

37. Wang DS, Zurek AA, Lecker I, et al. Memory deficits induced by inflammation are regulated by alpha5-subunit-containing GABAA receptors. Cell Rep. 2012;2:488-96.

38. Ikonomidou C, Bittigau P, Ishimaru MJ, et al. Ethanol-induced apoptotic neurodegeneration and fetal alcohol syndrome. Science. 2000;287:1056-60.

39. Olney JW, Ishimaru MJ, Bittigau P, et al. Ethanol-induced apoptotic neurodegeneration in the developing brain. Apoptosis. 2000;5:515-21.

40. Rice D, Barone S Jr. Critical periods of vulnerability for the developing nervous system: evidence from humans and animal models. Environ Health Perspect. 2000;108(suppl 3):511-33.

41. Dusica B, Commons KG, Soriano SG. Morphine-enhanced apoptosis in selective brain regions of neonatal rats. Int J Dev Neurosci. 2013;31(4):258-66.

42. Grady D. Researchers call for more study of anesthesia's risks to brains of young children. New York Times; 2015. p. A20.

43. Rappaport BA, Suresh S, Hertz S, et al. Anesthetic neurotoxicity-clinical implications of animal model. $\mathrm{N}$ Engl J Med. 2015;372:796-7.

44. Ben-Ari Y, Gaiarsa JL, Tyzio R, Khazipov R. GABA: a pioneer transmitter that excites immature neurons and generates primitive oscillations. Physiol Rev. 2007;87:1215-84.

45. Rudolph U, Antkowiak B. Molecular and neuronal substrates for general anaesthetics. Nat Rev Neurosci. 2004;5:709-20.

46. Nagele P, Metz LB, Crowder CM. Xenon acts by inhibition of non- $N$-methyl-D-aspartate receptor-mediated glutamatergic neurotransmission in Caenorhabditis elegans. Anesthesiology. 2005;103:508-13.

47. Young C, Jevtovic-Todorovic V, Qin YQ, et al. Potential of ketamine and midazolam, individually or in combination, to induce apoptotic neurodegeneration in the infant mouse brain. Br J Pharmacol. 2005;146:189-97.

48. Fredriksson A, Ponten E, Gordh T, et al. Neonatal exposure to a combination of $\mathrm{N}$-methyl-d-aspartate and gamma-aminobutyric acid Type A receptor anesthetic agents potentiates apoptotic neurodegeneration and persistent behavioral deficits. Anesthesiology. 2007;107:427-36.

49. Cattano D, Williamson P, Fukui K, et al. Potential of xenon to induce or to protect against neuroapoptosis in the developing mouse brain. Can J Anaesth. 2008;55:429-36.
50. Ma D, Williamson P, Januszewski A, et al. Xenon mitigates isoflurane-induced neuronal apoptosis in the developing rodent brain. Anesthesiology. 2007;106:746-53.

51. Yoshida M, Fukuda S, Tozuka Y, et al. Developmental shift in bidirectional functions of taurine-sensitive chloride channels during cortical circuit formation in postnatal mouse brain. J Neurobiol. 2004;60(2):166-75.

52. Kodama M, Satoh Y, Otsubo Y, et al. Neonatal desflurane exposure induces more robust neuroapoptosis than do isoflurane and sevoflurane and impairs working memory. Anesthesiology. 2011;115(5):979-91.

53. Bittigau P, Sifringer M, Genz K, et al. Antiepileptic drugs and apoptotic neurodegeneration in the developing brain. Proc Natl Acad Sci. 2002;99:15089-94.

54. Cattano D, Straiko MM, Olney JW. Chloral hydrate induces and lithium prevents neuroapoptosis in the infant mouse brain. Anesthesiology. 2008;109:A315.

55. Kahraman S, Zup SL, McCarthy MM, Fiskum G. Gabaergic mechanism of propofol toxicity in immature neurons. J Neurosurg Anesthesiol. 2008;20:233-40.

56. Sanders RD, Xu J, Shu Y, et al. Dexmedetomidine attenuates isoflurane-induced neurocognitive impairment in neonatal rats. Anesthesiology. 2009;110:1077-85.

57. Soriano SG, Liu J-R, Liu Q, et al. Dexmedetomidine is less proapoptotic than ketamine in the neonatal rat brain. Anesthesiology. 2009;A169.

58. Anand KJ, Sippell WG, Aynsley-Green A. Randomised trial of fentanyl anaesthesia in preterm babies undergoing surgery: effects on the stress response. Lancet. 1987;1(8527):243-8.

59. Lin CS, Tao PL, Jong YJ, et al. Prenatal morphine alters the synaptic complex of postsynaptic density 95 with $N$-methyl-Daspartate receptor subunit in hippocampal CA1 subregion of rat offspring leading to long-term cognitive deficits. Neuroscience. 2009;158:1326-37.

60. Istaphanous GK, Ward CG, Loepke AW. The impact of the perioperative period on neurocognitive development, with a focus on pharmacological concerns. Best Pract Res Clin Anaesthesiol. 2010;24:433-49.

61. Mintz CD, Wagner M, Loepke AW. Preclinical research into the effects of anesthetics on the developing brain: promises and pitfalls. J Neurosurg Anesthesiol. 2012;24:362-7.

62. Loepke AW, Soriano SG. An assessment of the effects of general anesthetics on developing brain structure and neurocognitive function. Anesth Analg. 2008;106:1681-707.

63. Terrando N, Monaco C, Ma D, et al. Tumor necrosis factoralpha triggers a cytokine cascade yielding postoperative cognitive decline. Proc Natl Acad Sci. 2010;107:20518-22.

64. Wilder RT, Flick RP, Sprung J, et al. Early exposure to anesthesia and learning disabilities in a population-based birth cohort. Anesthesiology. 2009;110:796-804.

65. Flick RP, Katusic SK, Colligan RC, et al. Cognitive and behavioral outcomes after early exposure to anesthesia and surgery. Pediatrics. 2011;128:e1053-61.

66. Sprung J, Flick RP, Wilder RT, et al. Anesthesia for cesarean delivery and learning disabilities in a population-based birth cohort. Anesthesiology. 2009;111:302-10.

67. DiMaggio CJ, Sun LS, Kakavouli A, et al. A retrospective cohort study of the association of anesthesia and hernia repair surgery with behavioral and developmental disorders in young children. J Neurosurg Anesthesiol. 2009;21:286-91.

68. Duncan GJ, Brooks-Gunn J. Consequences of growing up poor. New York: Russell Sage Foundation; 1997.

69. DiMaggio C, Sun L, Li G. Early childhood exposure to anesthesia and risk of developmental and behavioral disorders in a sibling birth cohort. Anesth Analg. 2011;113:1143-51. 
70. Sun LS, Li G, DiMaggio CJ, et al. Feasibility and pilot study of the pediatric anesthesia neuro development assessment (PANDA) project. J Neurosurg Anesthesiol. 2012;24(4):382-8.

71. Bartels M, Althoff RR, Boomsma DI. Anesthesia and cognitive performance in children: no evidence for a causal relationship. Twin Res Hum Genet. 2009;12:246-53.

72. Glatz P, Sandin RH, Pedersen NL, et al. Academic performance after anesthesia and surgery during childhood: a large-scale nation-wide study [abstract]. Int Anesth Res Soc: Anesth Analg. 2015;120(3)

73. Hansen TG, Pedersen JK, Henneberg SW, et al. Academic performance in adolescence after inguinal hernia repair in infancy: a nationwide cohort study. Anesthesiology. 2011;114:1076-85.

74. Hansen TG, Pedersen JK, Henneberg SW, et al. Educational outcome in adolescence following pyloric stenosis repair before 3 months of age: a nationwide cohort study. Paediatr Anaesth. 2013;23:883-90.

75. Ing $\mathrm{CH}$, DiMaggio CJ, Malacova E, et al. Comparative analysis of outcome measures used in examining neurodevelopmental effects of early childhood anesthesia exposure. Anesthesiology. 2014;120:1319-32.

76. Stratmann G, Lee J, Sall JW, et al. Effect of general anesthesia in infancy on long-term recognition memory in humans and rats. Neuropsychopharmacology. 2014;39(10):2275-87.

77. Backelijauw B, Holland SK, Altaye M, et al. Cognition and brain structure following early childhood surgery with anesthesia. Pediatrics. 2015;136(1):1-12.

78. Garcia Guerra G, Robertson CM, Alton GY, et al. Neurodevelopmental outcome following exposure to sedative and analgesic drugs for complex cardiac surgery in infancy. Paediatr Anaesth. 2011;21:932-41.

79. Garcia Guerra G, Robertson CM, Alton GY, et al. Neurotoxicity of sedative and analgesia drugs in young infants with congenital heart disease: 4-year follow-up. Paediatr Anaesth. 2014;24:257-65.

80. Andropoulos DB, Ahmad HB, Haq T, et al. The association between brain injury, perioperative anesthetic exposure, and 12-month neurodevelopmental outcomes after neonatal cardiac surgery: a retrospective cohort study. Paediatr Anaesth. 2014;24:266-74.

81. RozeÅL JC, Denizot S, Carbajal R, et al. Prolonged sedation and/or analgesia and 5-year neurodevelopment outcome in very preterm infants: results from the EPIPAGE cohort. Arch Pediatr Adolesc Med. 2008;162:728-33.

82. Davidson AJ, Disma N, deGraff JC, et al. Neurodevelopmental outcome at 2 years of age after general anaesthesia and awakeregional anaesthesia in infancy (GAS): an international multicenter, randomized controlled trial. Lancet. 2016;387(10015):239-50.

83. Consensus Statement on the Use of Anesthetic and Sedative Drugs in Infants and Toddlers [Internet], 2015. Available from: http://smarttots.org/about/consensus-statement/. Accessed 12 Nov 2015.

84. Riedel B, Browne K, Silbert B. Cerebral protection: inflammation, endothelial dysfunction, and postoperative cognitive dysfunction. Curr Opin Anaesthesiol. 2014;27(1):89-97.

85. Terrando N, Eriksson LI, Eckenhoff RG. Perioperative neurotoxicity in the elderly: summary of the 4th international workshop. Anesth Analg. 2015;120:649-52.

86. Silbert B, Evered L, Scott DA. Cognitive decline in the elderly: is anaesthesia implicated? Best Pract Res Clin Anaesthesiol. 2011;25:379-93.

87. Ramaiah R, Lam AM. Postoperative cognitive dysfunction in the elderly. Anesthesiol Clin. 2009;27:485-96.

88. Berger M, Nadler JW, Browndyke J, et al. Postoperative cognitive dysfunction: minding the gaps in our knowledge of a common postoperative complication in the elderly. Anesthesiol Clin. 2015;33(3):517-50.
89. Evered L, Scott DA, Silbert B, Maruff P. Postoperative cognitive dysfunction is independent of type of surgery and anesthetic. Anesth Analg. 2011;112:1179-85.

90. Moller JT, Cluitmans P, Rasmussen LS, et al. Long-term postoperative cognitive dysfunction in the elderly ISPOCD1 study. ISPOCD investigators. International Study of Post-Operative Cognitive Dysfunction. Lancet. 1998;351:857-61.

91. Abildstrom H, Rasmussen LS, Rentowl P, et al. Cognitive dysfunction 1-2 years after non-cardiac surgery in the elderly. ISPOCD group. International Study of Post-Operative Cognitive Dysfunction. Acta Anaesthesiol Scand. 2000;44:1246-51.

92. Newman MF, Kirchner JL, Phillips-Bute B, et al. Longitudinal assessment of neurocognitive function after coronary-artery bypass surgery. N Engl J Med. 2001;344:395-402.

93. Rudolf JL, Schreiber KA, Culley DJ, et al. Measurement of postoperative cognitive dysfunction after cardiac surgery: a systemic review. Acta Anaesthesiol Scand. 2010;54:663-77.

94. Wei H, Xie Z. Anesthesia, calcium homeostasis and Alzhemiers disease. Curr Alzheimer Res. 2009;6:30-5.

95. Xie Z, Dong Y, Maeda U, et al. The common inhalation anesthetic isoflurane induces apoptosis and increases amyloid beta protein levels. Anesthesiology. 2006;104:988-94.

96. Li C, Liu S, Xing Y, et al. The role of hippocampal tau protein phosphorylation in isoflurane-induced cognitive dysfunction in transgenic APP695 mice. Anesth Analg. 2014;119:413-9.

97. Wan Y, Xu J, Ma D, Zeng Y, Cibelli M, Maze M. Postoperative impairment of cognitive function in rats: a possible role for cytokine-mediated inflammation in the hippocampus. Anesthesiology. 2007;106:436-43.

98. Uchimoto K, Miyazaki T, Kamiya Y, et al. Isoflurane impairs learning and hippocampal long-term potentiation via the saturation of synaptic plasticity. Anesthesiology. 2014;121:302-10.

99. Li XM, Su F, Ji MH, et al. Disruption of hippocampal neuregulin 1-ErbB4 signaling contributes to the hippocampus-dependent cognitive impairment induced by isoflurane in aged mice. Anesthesiology. 2014;121:79-88.

100. Sall JW, Stratmann G, Leong J, et al. Isoflurane inhibits growth but does not cause cell death in hippocampal neural precursor cells grown in culture. Anesthesiology. 2009;110:826-33.

101. Hu N, Guo D, Wang H, et al. Involvement of the blood-brain barrier opening in cognitive decline in aged rats following orthopedic surgery and high concentration of sevoflurane inhalation. Brain Res. 2014;1551:13-24.

102. Bartels K, Ma Q, Venkatraman TN, et al. Effects of deep hypothermic circulatory arrest on the blood brain barrier in a cardiopulmonary bypass model-a pilot study. Heart Lung Circ. 2014;23(10):981-4.

103. Steinmetz J, Christensen KB, Lund T, Lohse N, Rasmussen LS, ISPOCD Group. Long-term consequences of postoperative cognitive dysfunction. Anesthesiology. 2009;110:548-55.

104. Tanzi RE. The synaptic Abeta hypothesis of Alzheimer disease. Nat Neurosci. 2005;8:977-9.

105. Tanzi RE. Tangles and neurodegenerative disease: a surprising twist. N Engl J Med. 2005;353:1853-5.

106. Tang J, Eckenhoff MF, Eckenhoff RG. Anesthesia and the old brain. Anesth Analg. 2010;110:421-6.

107. Baranov D, Bickler PE, Crosby GJ, et al. Consensus statement: first international workshop on anesthetics and Alzheimer's disease. Anesth Analg. 2009;108(5):1627-30.

108. Xie Z, Culley DJ, Dong Y, et al. The common inhalational anesthetic isoflurane induces caspase activation and increases amyloid beta-protein level in vivo. Ann Neurol. 2008;64:618-27.

109. Dong Y, Zhang G, Zhang B, et al. The common inhalational anesthetic sevoflurane induces apoptosis and increases beta amyloid protein levels. Arch Neurol. 2009;66:620-31. 
110. Bianchi SL, Tran T, Liu C, et al. Brain and behavior changes in 12-month-old $\mathrm{Tg} 2576$ and nontransgenic mice exposed to anesthetics. Neurobiol Aging. 2008;29:1002-10.

111. Culley DJ, Baxter M, Yukhananov R, Crosby G. The memory effects of general anesthesia persist for weeks in young and aged rats. Anesth Analg. 2003;96:1004-9.

112. Culley DJ, Baxter MG, Yukhananov R, Crosby G. Long-term impairment of acquisition of a spatial memory task following isoflurane-nitrous oxide anesthesia in rats. Anesthesiology. 2004;100:309-14.

113. Stratmann G, Sall JW, May LD, et al. Isoflurane differentially affects neurogenesis and long-term neurocognitive function in 60-day-old and 7-day-old rats. Anesthesiology. 2009;110:834-48.

114. Run X, Liang Z, Zhang L, et al. Anesthesia induces phosphorylation of tau. J Alzheimers Dis. 2009;16:619-26.

115. Planel E, Richter KE, Nolan CE, et al. Anesthesia leads to tau hyperphosphorylation through inhibition of phosphatase activity by hypothermia. J Neurosci. 2007;27:3090-7.

116. Degos V, Vacas S, Han Z, et al. Depletion of bone marrowderived macrophages perturbs the innate immune response to surgery and reduces postoperative memory dysfunction. Anesthesiology. 2013;118:527-36.

117. Tang JX, Mardini F, Janik L, et al. Modulation of murine Alzheimer pathogenesis and behavior by surgery. Ann Surg. 2013;257(3):439-48.

118. Wan Y, Xu J, Meng F, et al. Cognitive decline following major surgery is associated with gliosis, beta-amyloid accumulation and tau phosphorylation in old mice. Crit Care Med. 2010;38(11):2190-8.

119. Feng X, Degos V, Koch LG, et al. Surgery results in exaggerated and persistent cognitive decline in a rat model of the metabolic syndrome. Anesthesiology. 2013;118(5):1098-105.

120. Eckenhoff RG, Laudansky KF. Anesthesia, surgery, illness and Alzheimer's disease. Prog Neuropsychopharmacol Biol Psychiatry. 2012;47:162-6.

121. Tang W, et al. The growth factor progranulin binds to TNF receptors and is therapeutic against inflammatory arthritis in mice. Science. 2011;332(6028):478-84.

122. Cunningham C, Campion S, Lunnon K, et al. Systemic inflammation induces acute behavioral and cognitive changes and accelerates neurodegenerative disease. Biol Psychiatry. 2009;65:304-12.

123. Matteoli G, Gomez-Pinilla PJ, Nemethova A, et al. A distinct vagal anti-inflammatory pathway modulates intestinal muscularis resident macrophages independent of the spleen. Gut. 2014;63(6):938-48

124. Ye X, Lian Q, Eckenhoff MF, et al. Differential general anesthetic effects on microglial cytokine expression. PLoS One. 2013;8(1):e52887.

125. Yuki $\mathrm{K}, \mathrm{Bu} \mathrm{W}$, Shimaoka $\mathrm{M}$, et al. Volatile anesthetics, not intravenous anesthetic propofol bind to and attenuate the activation of platelet receptor integrin alphaIIbbeta3. PLoS One. 2013;8(4):e60415.

126. Fahlenkamp AV, Coburn M, Rossaint R, et al. Comparison of the effects of xenon and sevoflurane anaesthesia on leucocyte function in surgical patients: a randomized trial. Br J Anaesth. 2014;112(2):272-80.

127. Williams-Russo $P$, Sharrock NE, Mattis S, et al. Cognitive effects after epidural vs general anesthesia in older adults. A randomized trial. JAMA. 1995;274(1):44-50.

128. Mason SE, Noel-Storr A, Ritchie CW. The impact of general and regional anesthesia on the incidence of post-operative cognitive dysfunction and post-operative delirium: a systematic review with meta-analysis. J Alzheimers Dis. 2010;22(Suppl 3):67-79.

129. Tang N, Ou C, Liu Y, et al. Effect of inhalational anaesthetic on postoperative cognitive dysfunction following radical rectal resection in elderly patients with mild cognitive impairment. J Int Med Res. 2014;42(6):1252-61.

130. Bromander S, Anckarsater R, Kristiansson M, et al. Changes in serum and cerebrospinal fluid cytokines in response to nonneurological surgery: an observational study. J Neuroinflamm. 2012;9:242.

131. Ottens TH, Dieleman JM, Sauer AM, et al. Effects of dexamethasone on cognitive decline after cardiac surgery: a randomized clinical trial. Anesthesiology. 2014;121(3):492-500.

132. Chan MT, Cheng BC, Lee TM, et al. BIS-guided anesthesia decreases postoperative delirium and cognitive decline. J Neurosurg Anesthesiol. 2013;25:33-42.

133. Radtke FM, Franck M, Lendner J, et al. Monitoring depth of anaesthesia in a randomized trial decreases the rate of postoperative delirium but not postoperative cognitive dysfunction. $\mathrm{Br}$ J Anaesth. 2013;110(Suppl 1):i98-105.

134. Ballard C, Jones E, Gauge N, et al. Optimised anaesthesia to reduce post operative cognitive decline (POCD) in older patients undergoing elective surgery, a randomised controlled trial. PLoS One. 2012;7(6):e37410.

135. Whitlock EL, Villafranca AJ, Lin N, et al. Relationship between bispectral index values and volatile anesthetic concentrations during the maintenance phase of anesthesia in the B-Unaware trial. Anesthesiology. 2011;115(6):1209-18.

136. Gasparini M, Vanacore N, Schiaffini C, et al. A case-control study of Alzheimer's disease and exposure to anesthesia. Neurol Sci. 2002;23:11-4.

137. Bohnen N, Warner MA, Kokmen E, Kurland LT. Early and midlife exposure to anesthesia and the age of onset of Alzheimer's disease. Int J Neurosci. 1994;77:181-5.

138. Avidan MS, Searleman AC, Storandt M, et al. Long-term cognitive decline in older subjects was not attributable to noncardiac surgery or major illness. Anesthesiology. 2009;11:964-70.

139. Ehlenbach WJ, Hough CL, Crane PK, et al. Association between acute care and critical illness hospitalization and cognitive function in older adults. JAMA. 2010;303:763-70.

140. Chen CW, Lin CC, Chen KB, et al. Increased risk of dementia in people with previous exposure to general anesthesia: a nationwide population-based case-control study. Alzheimers Dement. 2014;10(2):196-204.

141. Chen PL, Yang CW, Tseng YK, et al. Risk of dementia after anaesthesia and surgery. Br J Psychiatry. 2014;204(3):188-93.

142. Kline RP, Pirraglia E, Cheng H, et al. Surgery and brain atrophy in cognitively normal elderly subjects and subjects diagnosed with mild cognitive impairment. Anesthesiology. 2012;116(3): 603-12.

143. Sprung J, Jankowski CJ, Roberts RO, et al. Anesthesia and incident dementia: a population-based, nested, case-control study. Mayo Clin Proc. 2013;88(6):552-61.

144. Moller JT, Cluitmans P, Rasmussen LS, et al. Long-term postoperative cognitive dysfunction in the elderly ISPOCD1 study. ISPOCD investigators. International Study of Post-Operative Cognitive Dysfunction. Lancet. 1998;351(9106):857-61.

145. Evered L, Silbert B, Ames D, et al. Cerebrospinal fluid biomarker for Alzheimer disease predicts postoperative cognitive dysfunction. Anesthesiology. 2016;124(2):353-61.

146. Xie Z, McAuliffe S, Swain CA, et al. Cerebrospinal fluid a $\beta$ to tau ratio and postoperative cognitive change. Ann Surg. 2013;258(2):364-9. 